\title{
A Soft Multi-Axis Force Sensor
}

\author{
Daniel Vogt, Yong-Lae Park and Robert J. Wood
}

\begin{abstract}
Thin, highly compliant sensing skins could provide valuable information for a host of grasping and locomotion tasks with minimal impact on the host system. We describe the design, fabrication and characterization of a novel soft multi-axis force sensor made of highly deformable materials. The sensor is capable of not only measuring normal force and but also 2D in-plane forces. This soft sensor is composed of an elastomer (modulus: $69 \mathrm{kPa}$ ) embedded with microchannels filled with conductive liquid. Depending on the intensity and the direction of an applied force, the cross-section of some of the channels will be compressed, changing its electrical resistance. The channel dimensions of the current prototype are $200 \mu \mathrm{m} \times$ $200 \mu \mathrm{m}$ and the overall size of the sensor is $50 \mathrm{~mm} \times 60 \mathrm{~mm} \times$ $7 \mathrm{~mm}$. Characterization results showed sensitivities in the two principal in-plane directions of $37.0 \mathrm{mV} / \mathrm{N}$ and $-28.6 \mathrm{mV} / \mathrm{N}$, respectively.
\end{abstract}

\section{INTRODUCTION}

The development of soft sensors is a key challenge in the domains of soft robotics [1], wearable electronics [2], haptics interfaces [3], robot manipulators [4], humanoid robotics [5], medical robotics [6], [7] and many more. In addition to being soft (modulus $<1 \mathrm{MPa}$ ), soft sensors must be durable, impact resistant, remain electronically functional when stretched and composed of relatively low-cost raw materials.

One example of such sensors is an artificial skin made of an elastomer embedded with microchannels filled with a conductive liquid [8]. When pressure is applied, the cross-sectional area of nearby microchannels is reduced, increasing their electrical resistance. Using this principle, properties such as stress [8], normal surface pressure [9] and curvature [10] can be measured using only soft or liquidphase materials. However, these sensors are not capable of detecting in-plane (i.e. shear) forces. In this work, the same principle is used to extend the capability of soft sensors to detect in-plane forces.

Various rigid and flexible Microelectromechanical systems (MEMS) based force and shear sensors [11] [12] have been reported for applications such as fluid dynamic monitoring [13] and above-knee prosthesis [14]. A flexible multi-axis sensor using a "dome" configuration and strain gauges has been proposed in [15]. However, these sensors do not provide high elasticity and deformability that are critical properties of soft robots.

In this paper, we present a novel design for a soft multiaxis force sensor and describe the operating principle and

All authors are with the Harvard Microrobotics Laboratory, http://micro.seas.harvard.edu School of Engineering and Applied Sciences, Wyss Institute for Biologically Inspired Engineering, Harvard University, Cambridge, MA, USA. (e-mails: dvogt@seas.harvard.edu, ylpark@wyss.harvard.edu, and rjwood@eecs.harvard.edu)

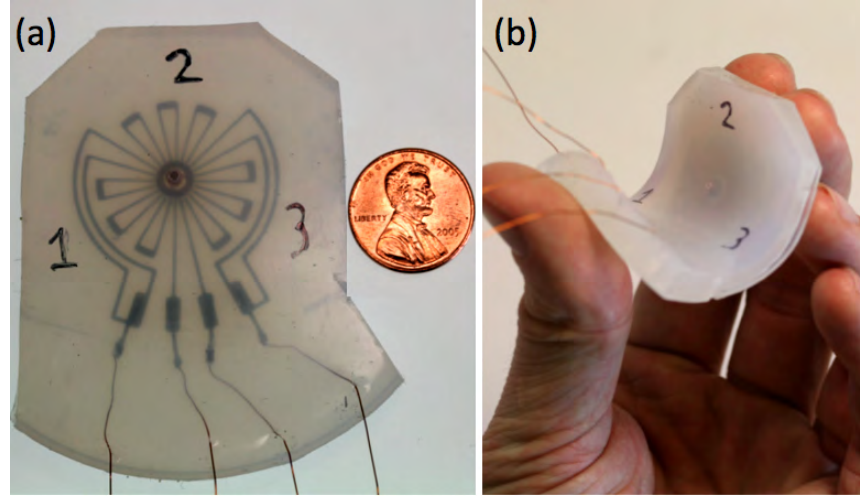

Fig. 1. Prototype of the soft multi-axis force sensor.

fabrication method. We also provide experimental characterization results. The sensor exhibits significant resistance changes for in-plane forces in the range of 0 to $1.1 \mathrm{~N}$ and for normal forces from 0 to $4.5 \mathrm{~N}$.

The proposed sensor can be an ideal component of artificial skins for robot manipulators and locomotive robots, where a highly compliant sensing skin would provide valuable proprioceptive or exteroceptive (e.g. tactile) information with minimal impact on the existing motion of the host.

\section{DESIGN}

An elastomer-based sensor (Fig. 1) was developed to measure three axis forces (normal and in-plane forces) on its surface. The sensor contains microchannels filled with a liquid metal (eutectic Gallium Indium, eGaIn) and a rigid plastic force-post.The force-post is prototyped using a $3 \mathrm{D}$ printer ${ }^{1}$ before being embedded in a silicone rubber substrate $^{2}$.

The sensor is composed of two layers as shown in Fig. 2(a). The top layer contains the force-post and the bottom layer contains the eGaIn microchannels patterned with a rotational symmetry, as shown in in Fig. 3. Since we desire three-axis force sensing, we need a minimum of three sensing elements. By arranging three sensing elements in a star pattern, we can decipher in-plane forces in two axes and a normal force.The three channels are separated by $120^{\circ}$ and intersect under the force-post. For the sake of wiring, the channels extend out to the perimeter of the sensor. Although the minimum number of sensing elements is three, the addition of more channels can increase the sensor accuracy at the expense of spatial complexity.

${ }^{1}$ Connex 500, Objet Geometries, Billerica, MA 01821, USA.

${ }^{2}$ EcoFlex0030, Smooth-On Inc., Easton, PA 18042, USA. 
(a)

(b)

(c)

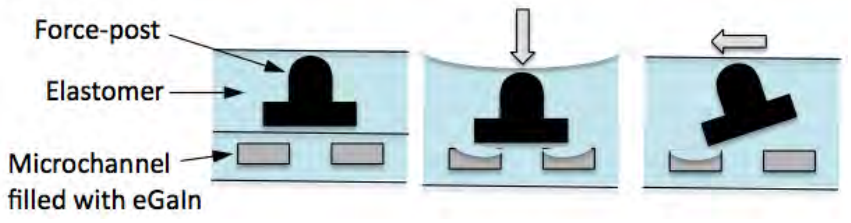

Fig. 2. (a) Description of the different layers of the sensor. (b) Deformation due to a pressure (c) Deformation due to an in-plane force. (a)

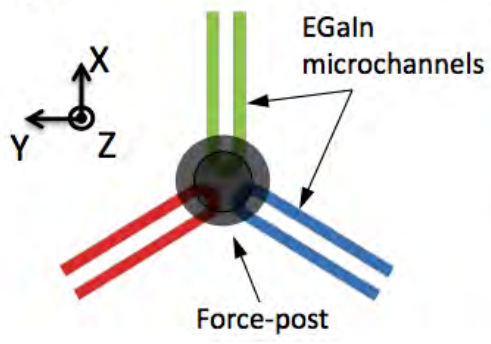

(b)

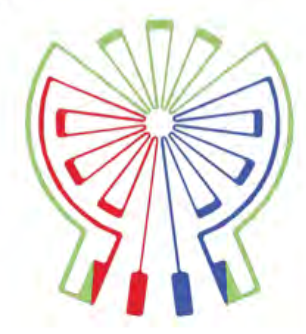

Fig. 3. (a) Simplified representation of the star configuration of the eGaIn microchannels. (b) Current design of the microchannels.

Fig. 3 (a) shows a simplified representation of the starshaped microchannel pattern along with the final configuration. The design is compatible with our fabrication process where eGaIn is injected into the channels; the geometry in Fig. 3(b) combines the three sensing elements into one channel, thus facilitating filling.

In addition, although our channel design, shown in Fig. 3(b), is electrically equivalent to the simplified configuration in Fig. 3(a), the microchannles in our design pass underneath the force-post several times, thereby increasing the overall sensitivity. The microchannels can be divided into three major channels, with each channel having eight sub channels, for a total of 24 channels under the force-post.

When a normal force is applied on the surface, the forcepost compresses all channels, resulting in increased electrical resistance three channels (Fig. 2(b)). With an in-plane force, the force-post will rotate and press only one or two of the three microchannels (Fig. 2 (c)) depending on the configuration of the channels, which also causes increased resistance of the deformed channels. By monitoring the resistance changes of the three sensor signals, we can determine the direction ( $x, y$, or $z$ axis) and magnitude of the force.

\section{FABRICATION}

The fabrication steps for the sensor prototype are represented in Fig. 4. First, the microchannels are fabricated by pouring liquid silicone rubber in two 3D printed molds (Fig. 4 (a)), one for casting a microchannel patterned top layer and the other for a flat bottom layer. Once cured, these two layers are bonded together (Fig. 4 (c)) by spin coating (2000 rpm, $50 \mathrm{sec}$, Fig. 4 (b)) a thin layer of uncured elastomer. The microchannels are then filled with eGaIn in the two middle reservoirs using two syringes (Fig. 4 (d)). While one of the (a)
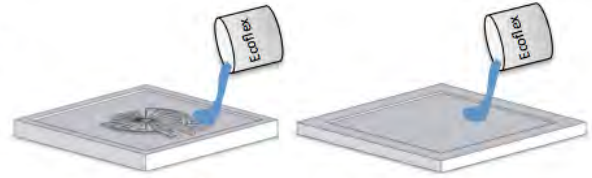

(b)

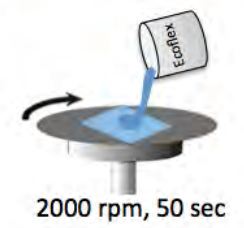

(d)
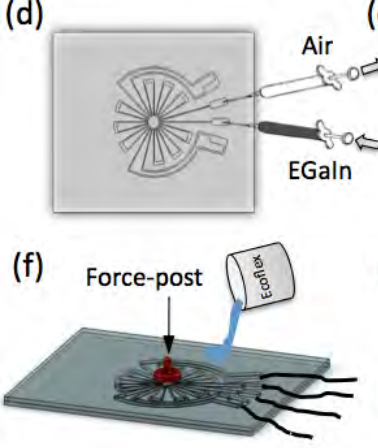

(c)

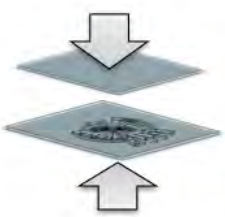

(e)

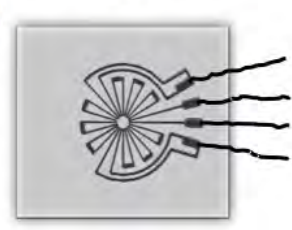

(g)

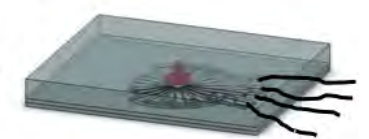

Fig. 4. Fabrication process : (a) Pour ecoflex in the molds, (b) Spin coat a thin layer of EcoFlex on the flat surface, (c) Bond the 2 layers, (d) Inject eGaIn using two syringes, (e) Insert wires, (f) Place the force-post and pour EcoFlex, (g) Finished multi-axis force sensor.

syringes injects eGaIn, the other evacuates the air captured in the channel during layer bonding process. Electrical connections are then made using thin wires inserted into each reservoir (Fig. 4 (e)). The force-post is then placed (Fig. 4 (f)), and Ecoflex is cast as an upper layer until the force-post is completely covered (Fig. $4(\mathrm{~g})$ ).

\section{EXPERIMENTAL SETUP}

By applying a constant current source in series with all three channels, changes in resistance from applied pressures can be read as voltage changes. In order to characterize the sensitivity of the sensors, we describe an experimental setup that enables us to apply a known load and read the resulting voltage change.

\section{A. Test Bench Integrated with a Multi-Axis Force Sensor}

To characterize the sensor, we first need to apply a known load. This can be achieved by mounting a 6-axis force and torque sensor to a 3-axis translation stage. Fig. 5 (a) shows the translation stage ${ }^{3}$ chosen for this purpose. This translation stage has a travel range of $9.5 \mathrm{~mm}$ and a load capacity of $13.3 \mathrm{~N}$.

A custom interface connects the commercial multi-axis force sensor ${ }^{4}$ to the 3 -axis translation stage. The force sensor is then connected to a data acquisition PCI $\operatorname{card}^{5}$ on a PC, and the data can be processed in Matlab ${ }^{\circledR}$.

${ }^{3}$ M-MT-XYZ, Newport, North Billerica, MA 01862, USA.

${ }^{4}$ F/T Nano 17, ATI Industrial Automation, Apex, NC 27539 USA.

${ }^{5}$ NI-6221, National Instruments, Austin, TX 78759, USA. 
(a)

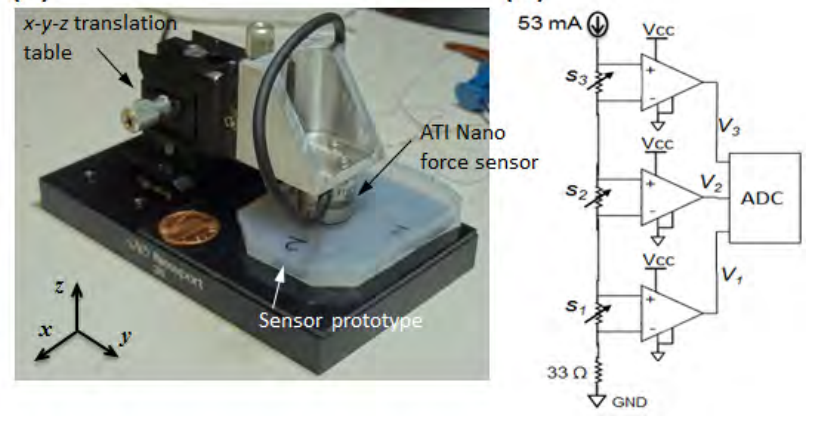

Fig. 5. (a) Translation stage mounted with a force sensor. (b) Schematic of the electronic circuit. S1, S2 and S3 corresponding to the variable resistance of the soft multi-axis force sensor.

\section{B. Amplifier Circuit}

In order to evaluate the force values, the soft sensor's output are first connected to an amplifier circuit (Fig. 5 (b)). The circuit first provides constant current through variable resistances of the soft sensor. The voltages across each channel are amplified (46×) using an op-amp. The amplified voltages are read by a data acquisition tool ${ }^{6}$ and data can be read using Matlab ${ }^{\circledR}$.

\section{Sensor Characterization}

To characterize the soft sensor, varied forces are applied in the $x, y$, and $z$ directions as defined in Fig. 5 using the translation stage and the multi-axis force/torque sensor. The force sensor is in direct contact with the top surface of the soft sensor. To prevent the two surfaces from sliding with respect to each other while applying in-plane forces, a thin layer of sandpaper was firmly attached to the surface of the commercial sensor.

To record the force value as well as the sensor's output signals, a Matlab ${ }^{\circledR}$ GUI was written that allows the user to see real-time forces applied. Data is sampled at $100 \mathrm{~Hz}$, and each data point is the average of the most recent 50 samples. We perform two tests, measuring sensor output for normal forces and two axes of lateral forces. Note that this is only a static characterization of the sensor.

\section{A. Sensor Output vs. Normal Force}

When applying a normal force, the force-post compresses all three microchannels evenly, and the resistances of all three microchannels exponentially increase, as shown in [9].

In this experiment, we gradually increased the force in the $z$-axis from $0 \mathrm{~N}$ to $6 \mathrm{~N}$, then also gradually release the force back to zero to evaluate hysteresis. The result of this experiment is shown in Fig. 6(a).

\section{B. Sensor Output vs. In-Plane Forces}

To measure in-plane forces, a pre-force normal to the sensor's surface is required in order to avoid slip between the two surfaces. An initial vertical force of $2 \mathrm{~N}$ is applied

\footnotetext{
${ }^{6}$ USB-1208LS, Measurement Computing, Norton, MA 02766, USA.
}

and regulated throughout the experiment. Then, we translate the force sensor along either the $x$ or $y$ axes and measure the resulting force signals. Fig. 6(b, c) shows a diagram of these tests and the resulting soft sensor responses. As with normal force characterization, the reference force sensor is brought back to its original position after applying both positive and negative force to evaluate hysteresis.

\section{Results AND Discussion}

The experiments described in Sections V-A and V-B fully characterize the static performance of the sensor. As shown in Fig. 6, there is slight hysteresis in the sensor. This may be due to the constraints applied by our experimental setup: the experiments described in sec. $\mathrm{V}$ consist of position control of the commercial force sensor in contact with the soft force sensor. Therefore, it is not surprising that we do not see significant hysteresis in the sensor signals. If the surface of the sensor was free, we would expect to see some hysteresis due to the visco-elastic nature of the elastomer materials. However, given the scope of applications we envision where objects are in constant direct contact with the sensor - we believe that the characterization in Section V is most appropriate.

As shown in Fig. 6 (a), the response to normal force displays an exponential response. This is expected due to the reduction in cross-section of the channel containing the liquid metal as previously shown in [9]. It is important to also notice that the three sensor responses are not exactly the same. This may be due to slight differences in the channel geometries and in the wire connections. In addition, the current design makes the length of the S2 channel slightly longer than the other two, resulting in a higher nominal resistance.

As can be seen in Fig. $6(b, c)$, the response to inplane force shows strong directional sensitivity. For example, positive force along the $\mathrm{x}$-axis is detected by an increase in S2 and a simultaneous decrease in S1 and S3. The offset between S1 and S3 is likely due to the difference in the response to the initial pressure.

When applying force along the $y$ axis, $\mathbf{S} 1$ and $\mathbf{S} 3$ change symmetrically as one can expect. S2 stays at its initial level as the applied force is perpendicular to its sensitivity axis.

Using a linear fitting, we can estimate the in-plane force sensitivity of $37.0 \mathrm{mV} / \mathrm{N}$ for the $x$ axis and $-28.6 \mathrm{mV} / \mathrm{N}$ for the $y$ axis (with S1). Due to its exponential response, the sensitivity to pressure varies from $27.8 \mathrm{mV} / \mathrm{N}$ for forces bellow $3.4 \mathrm{~N}$ and $572.7 \mathrm{mV} / \mathrm{N}$ for pressures over $4.1 \mathrm{~N}$ (S2).

\section{CONClusion AND Future Work}

This study describes the design, fabrication, and characterization of a novel soft multi-axis force sensor. This sensor can not only detect the normal force to the top surface but also measure the direction and magnitude of an applied inplane (i.e. shear) force.

Future work will expand our understanding of the different parameters composing the soft force sensor. Example parameters include the geometry of the force-post (height 


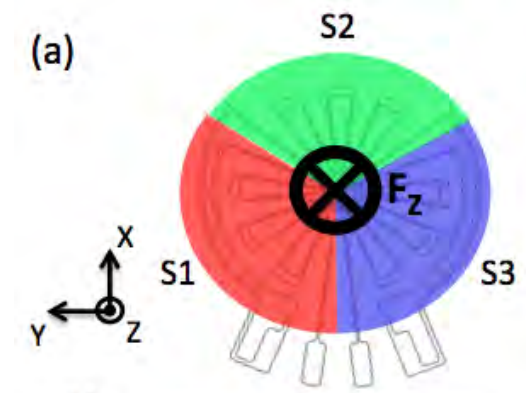

(b)
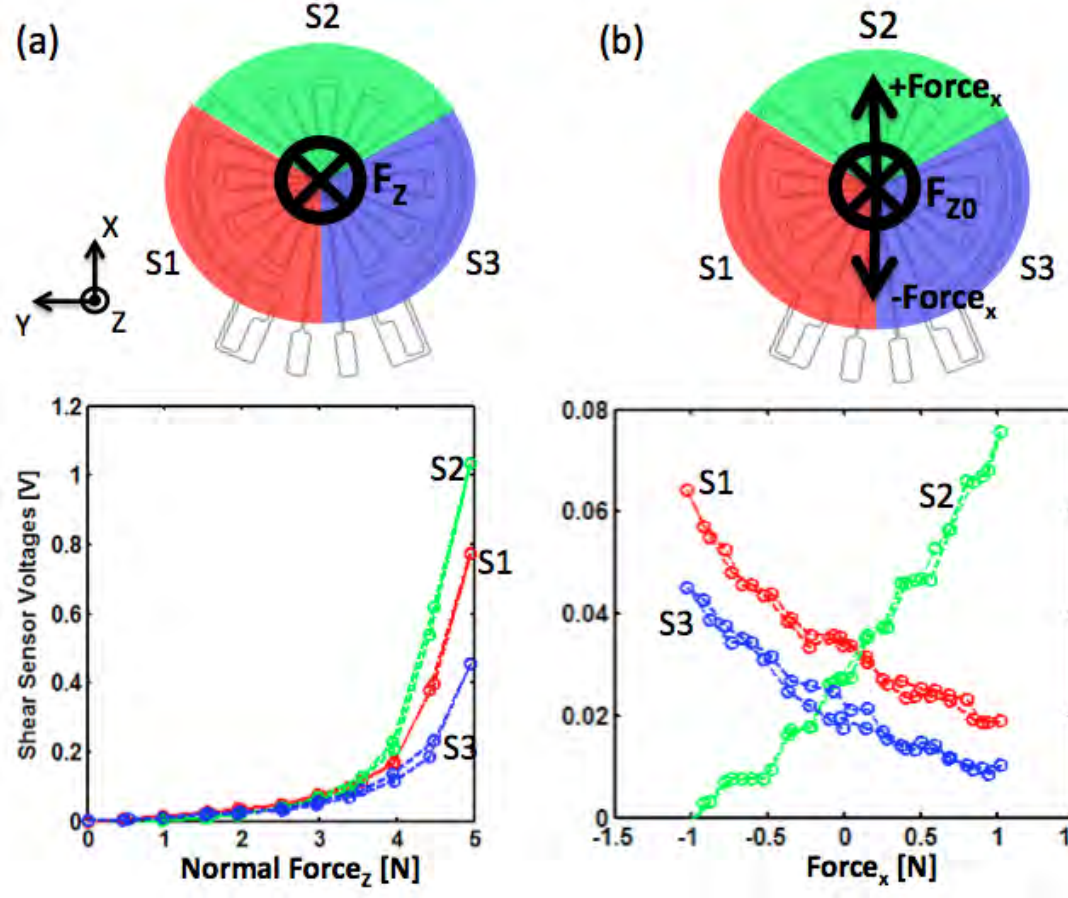

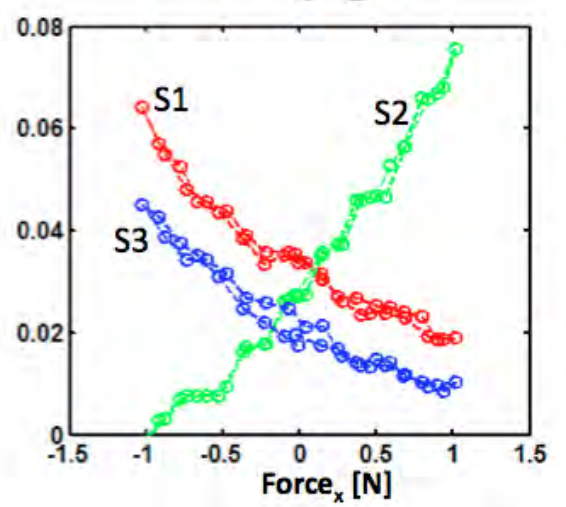

(c)

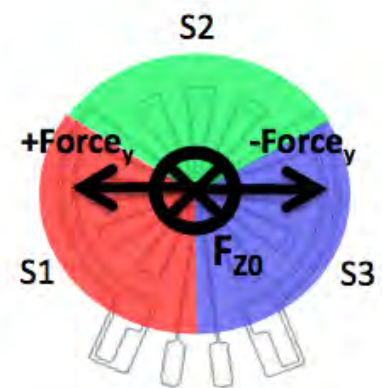

Fig. 6. Characterization Results : (a) Output voltages vs. normal force. (b) Output voltages vs. force along the x axis. (c) Output voltages vs. force along the $y$ axis.

and diameter), the size of the microchannels and the total number of microchannels.

For example, to increase the sensor accuracy, a variant will be designed with four channels separated by $90^{\circ}$ instead of three separated by $120^{\circ}$ for a better estimation of the in-plane force direction.

We will also reduce the thickness (currently $7 \mathrm{~mm}$ ) of the sensor using photolithography techniques to create molds as described in [9].

Finally, the sensor can be fitted to a robotic hand to test grasping with closed-loop force control. The idea will be to lift an object, using minimal normal force. We speculate that, with the in-plane force information, the robot will be able to determine the quality of a static grasp and also sense slipping and grasp failure.

\section{REFERENCES}

[1] Y.-L. Park, B. Chen, D. Young, L. Stirling, R. J. Wood, E. Goldfield, and R. Nagpal, "Bio-inspired active soft orthotic device for ankle foot pathologies," in Proc. IEEE/RSJ Int. Conf. Intell. Rob. Syst., (San Francisco, CA), September 2011.

[2] D. Marculescu, R. Marculescu, N. Zamora, P. Stanley-Marbell, P. Khosla, S. Park, S. Jayaraman, S. Jung, C. Lauterbach, W. Weber, et al., "Electronic textiles: A platform for pervasive computing," Proceedings of the IEEE, vol. 91, no. 12, pp. 1995-2018, 2003.

[3] A. Okamura and M. Cutkosky, "Feature detection for haptic exploration with robotic fingers," The International Journal of Robotics Research, vol. 20, no. 12, pp. 925-938, 2001.

[4] Y.-L. Park, S. C. Ryu, R. J. Black, K. Chau, B. Moslehi, and M. R. Cutkosky, "Exoskeletal force-sensing end-effectors with embedded optical fiber-bragg-grating sensors," IEEE Trans. Rob., vol. 25, pp. 13191331, December 2009.

[5] R. Tajima, S. Kagami, M. Inaba, and H. Inoue, "Development of soft and distributed tactile sensors and the application to a humanoid robot," Advanced Robotics, vol. 16, no. 4, pp. 381-397, 2002.
[6] P. Puangmali, K. Althoefer, L. Seneviratne, D. Murphy, and P. Dasgupta, "State-of-the-art in force and tactile sensing for minimally invasive surgery," Sensors Journal, IEEE, vol. 8, no. 4, pp. 371-381, 2008.

[7] Y.-L. Park, S. Elayaperumal, B. Daniel, S. C. Ryu, M. Shin, J. Savall, R. J. Black, B. Moslehi, and M. R. Cutkosky, "Real-time estimation of 3-d needle shape and deflection for mri-guided interventions," IEEE/ASME Trans. Mechatron., vol. 15, pp. 906-915, December 2010.

[8] Y.-L. Park, B. Chen, and R. J. Wood, "Design and manufacturing of soft artificial skin using embedded microchannels and liquid conductors," IEEE Sens. J., vol. 12, no. 8, pp. 2711-2718, 2012.

[9] Y.-L. Park, C. Majidi, R. Kramer, P. Berard, and R. J. Wood, "Hyperelastic pressure sensing with a liquid-embedded elastomer," $J$. Micromech. Microeng., vol. 20, no. 12, 2010.

[10] C. Majidi, R. Kramer, and R. Wood, "A non-differential elastomer curvature sensor for softer-than-skin electronics," Smart Materials and Structures, vol. 20, p. 105017, 2011.

[11] A. Barlian, S. Park, V. Mukundan, and B. Pruitt, "Design and characterization of microfabricated piezoresistive floating element-based shear stress sensors," Sensors and Actuators A: Physical, vol. 134, no. 1, pp. 77-87, 2007.

[12] F. Jiang, Y. Tai, K. Walsh, T. Tsao, G. Lee, and C. Ho, "A flexible mems technology and its first application to shear stress sensor skin," in Micro Electro Mechanical Systems, 1997. MEMS'97, Proceedings, IEEE., Tenth Annual International Workshop on, pp. 465-470, IEEE, 1997.

[13] Y. Xu, Y. Tai, A. Huang, and C. Ho, "IC-integrated flexible shear-stress sensor skin," Microelectromechanical Systems, Journal of, vol. 12, no. 5, pp. 740-747, 2003.

[14] M. Hsieh, Y. Fang, M. Ju, G. Chen, J. Ho, C. Yang, P. Wu, G. Wu, and T. Chen, "A contact-type piezoresistive micro-shear stress sensor for above-knee prosthesis application," Microelectromechanical Systems, Journal of, vol. 10, no. 1, pp. 121-127, 2001.

[15] H. Kwon, J. Kim, and W. Choi, "Development of a flexible three-axial tactile sensor array for a robotic finger," Microsystem Technologies, pp. 1-6, 2011. 